\title{
THE STUDY OF SOCIAL-MEDICAL AND GENETIC RISKS OF REPRODUCTIVE LOSSES
}

\author{
Alieva Tarana Dzhafar Kyzy ${ }^{1}$ \\ ${ }^{1}$ Department of Public Health and Health Care Management, Ukrainian Institute of Clinical Genetics, Kharkiv National Medical University, \\ Kharkiv, Ukraine \\ alieva_tdk@ukr.net \\ http://orcid.org/0000-0002-3002-667X
}

ARTICLE INFO

ABSTRACT

Article history:

Received date 19.01.2021

Accepted date 17.02.2021

Published date 22.02.2021

Object of research: homozygous and heterozygous disorders of genes encoding the en-

Section:

Practical medicine zymes of the folate cycle, methylenetetrahydrofolate reductase $(M T H F R)$ and methionine-synthase-reductase (MTRR).

DOI

Solved problem: an in-depth study of genetically determined risk factor's influence for reproductive losses associated with homozygous and heterozygous disorders of folate cycle genes.

$10.21303 / 2313-8416.2021 .001668$ Main scientific results: in-depth study of genetically determined reproductive losses as a systemic phenomenon was held. The structural characteristics of reproductive losses in population and significant predominance of pathology in the pedigrees of those examined

KEYWORDS with a burdened obstetric history of reproductive losses were determined. Also, a signifi-

reproductive population loss

fetal malformations cant increase in the chances of reproductive loss in patients with heterozygous and homo-

gene polymorphism

folate cycle

MTHFR C677T

MTRR A66G zygous inheritance of MTHFR and MTRR genes was determined. A correlation effect on the degree of genomic polymorphism of the MTHFR and MTRR gene was noted.

Area of practical use of research results: medical-genetic institutions.

Innovative technological product: determination of genetically risk factors for growth of reproductive losses of the population associated with homozygous and heterozygous disorders of genes encoding the enzymes of the folate cycle (MTHFR C677T and MTRR A66G). Timely adjustment of folic acid levels allows to prevent birth defects and reduce reproductive losses

Scope of application of the innovative technological product: clinical medical-genetic practice using the ability to determine the polymorphism of genes MTHFR and MTRR, which makes it possible to timely adjust the level of folic acid and prevent the reproductive losses.

(C) The Author(s) 2021. This is an open access article under the CC BY license http://creativecommons.org/licenses/by/4.0).

\section{Introduction}

\section{1. Object of research}

Homozygous and heterozygous disorders of genes encoding folate cycle enzymes: methylenetetrahydrofolate reductase (MTHFR C677T) and methionine-synthase-reductase (MTRR A66G).

\section{2. Problem description}

Modern development of molecular genetic technologies allows timely to diagnose hereditary diseases, which cause a significant part of reproductive losses (RL). And new global paradigms of medicine (Predictive, Personalized, Preventive and Participatory, or 4P) help to build a multilevel preventive system of hereditary genetic pathology, which can positively affect the level of RL in the early stages of ontogenesis (pregametic, presygotic, embryonic and fetal) [1, 2]. For the solution of the whole task, the etiological factors and diagnostic markers for the destruction of human embryogenesis and development of the fetus (biochemical, molecular-genetic, cytogenetic, instrumental, etc.) permanently studied in all sides of the world.

Despite of the scientific researches' successes over the past decades, a lot of RL remain with unexplained etiology. High rates of miscarriage and life-threatening birth defects depend on genetic factors $[3,4]$. This fact confirms the relevance and significance of our research.

\section{3. Suggested solution to the problem}

Among all RL, genetic caused are most significant (16-55\%) [5]. Other important causes are anatomical (5-29\%), infectious (2-45\%), endocrine (15-40\%), and immunological (up to 
$40 \%$ ). Another 20-40\% RLs remain unclear. The study of pathology of pregnancy and fetal development associated with RL [6], led to a clear understanding of the causal relationship between disorders of the amino acids homocysteine and methionine in the blood, folic acid deficiency and polymorphism of genes encoding folate cycle (FC) enzymes [7, 8].

An in-depth study of the genes encoding the FC enzymes, namely methylenetetrahydrofolate reductase (MTHFR C677T) and methionine-synthase-reductase (MTRR A66G), was to study the relationship between homozygous and heterozygous types of inheritance, the strength and influence of their clinical manifestations of FC violations.

The aim of research includes study of the influence of social-medical and genetic risk factors on the level of reproductive losses, taking into account the strength of clinical manifestations of defective folate genes with different types of inheritance.

\section{Materials and methods of research}

In accordance with the purpose and objectives of our study, during 2011-2013, 154 patients of the diagnostic group (DG) with RL in anamnesis, and 160 patients of the control group (CG; without RL in anamnesis) were prospectively examined. All patients asked preconception care for planning future pregnancies into the Municipal non-Commercial Enterprise of Kharkiv Regional Council "Inter-Regional Specialized Medical-Genetic Center - Center of Rare (Orphanic) Diseases" (MNCE KhRC “IRSMGC-CR(O)D”) for planning future pregnancies and conducting appropriate preconception care. DG patients were examined cytogenetically. According to the result, they were divided into 2 subgroups: DG1 (56 persons) - with a normal karyotype, and DG2 (98 persons) - with a pathologically altered karyotype. Patient data is kept confidential. Patients were notified to participate in the study (written informed consent was obtained). The study was approved by the Bioethics Committee of Kharkiv National Medical University (protocol No. 6 dated October 03, 2011) as conducted in accordance with the WMA Declaration of Helsinki - Ethical Principles for Medical Research Involving Human Subjects, revised in Fortaleza, Brazil, October 2013).

The calculation of the correlation coefficient was performed according to the formula:

$$
r=\frac{\sum d_{x} \times d_{y}}{\sqrt{\sum d_{x}^{2} \times \sum d_{y}^{2}}},
$$

where $x$ and $y$ - variants of variation series comparing; $d_{x}$ and $d_{y}-$ deviation of each variant from its arithmetic mean.

The nature of the relationship between these parameters was determined using the rank correlation coefficient $(r)$. Correlation was studied by the direction, strength, and form of the relationship. The strength of the connection was assessed on a scale:

$$
\begin{aligned}
& -r=0.01-0.29 \text { (weak connection); } \\
& -r=0.3-0.69 \text { (medium connection); } \\
& -r=0.7-0.99 \text { (strong connection). }
\end{aligned}
$$

During the statistical processing of the obtained results the methods of calculation of relative and average values, estimation of their reliability, stratification and correlation-regression analysis were used. Maintenance of the study data bank, basic calculations of derivative indicators, frequency response of characteristics were performed using Microsoft Excel 2016 software (license No. 00201-10554-16848-AA351), all calculations were performed using Statsoft Statistica 8.0 (license No. STA862D175437Q). In addition, the results of cytological examination of 6824 patients during the period 2008-2013 in the MNCE KhRC "IRSMGC-CR(O)D” were retrospectively studied.

\section{Results}

According to the results of a prospective study of 154 patients from DG, RL cases in anamnesis, which were represented by miscarriages, missed abortion, antenatal death, early neonatal death, ectopic pregnancies, multiple fetal birth defects, incompatible with life, were identified. The spectrum of these violations is shown in Table 1. The age of DG and CG patients ranged from 19 to 47 years; the average age of DG patients was 29.9 years, $C G-28.6$ years. CG patients asked for 
routine examination and pregnancy planning into MNCE KhRC "IRSMGC-CR(O)D”, and did not have a burdensome obstetric anamnesis.

Table 1

RL varieties in patients from DG with a burdensome obstetric anamnesis (absolute value; \%)

\begin{tabular}{ccc}
\hline RL in anamnesis & Absolute val. & \% \\
\hline One miscarriage & 23 & 14.94 \\
Two or more miscarriages & 19 & 12.34 \\
One ectopic pregnancy & 8 & 5.19 \\
Two ectopic pregnancies & 1 & 0.65 \\
Missed abortion & 41 & 26.62 \\
Antenatal fetal death & 8 & 5.19 \\
Early neonatal death & 3 & 1.95 \\
Multiple fetal birth defects, incompatible with life & 1 & 0.65 \\
Secondary infertility & 7 & 4.55 \\
Various RL combinations & 43 & 27.92 \\
Total & 154 & 100.00
\end{tabular}

According to the study, most of DG patients had cases of one miscarriage (14.94\%), less often - two or more miscarriage $(12.34 \%)$. More than one from four women $(26.62 \%)$ had missed abortion, antenatal fetal death $-5.19 \%$, ectopic pregnancy $-5.19 \%$, two ectopic pregnancies $0.65 \%$, secondary infertility $-4.55 \%$, early neonatal fetal death $-1.95 \%$, multiple fetal birth defects, incompatible with life $-0.65 \%$. Combinations of different RL nosology were noted in every third (27.92\%) patient with DG, which proves the validity of an in-depth study of genetically determined RL etiology as a systemic phenomenon.

When comparing the results of cytogenetic examination of women of subgroups DG1 (with normal karyotype) and DG2 (with pathologically altered karyotype) with genealogical data of patients of all groups, special attention to the presence of reproductive disorders in blood relatives of patients, cardiovascular and oncology diseases was paid. It was established whether any of the relatives of the patients were registered with geneticists or other physicians. The structure of the revealed pathology in the pedigree of the examined patients is shown by Fig. 1.

The identified cases of all nosological forms predominated in DG patients. Thus, in the pedigrees of DG1 patients chromosomal pathology was recorded in $5.4 \%$ cases (every eighteenth patient), DG2 - in $13.3 \%$ cases (every seventh patient), and among patients CG was not detected. Among the identified cases of chromosomal pathology were two cases of Down syndrome, one of Klinefelter's syndrome. All cases of fetal polyploidy were detected using invasive methods of prenatal diagnosis. Three cases of missed abortion in DG1 patients were identified as a result of chorionic biopsy. Gene pathology among relatives of patients of all groups was less compared to chromosomal: in CG - 3.1\%; DG1 - 7.1\%; DG2 - 8.2\%. Multifactorial pathology in pedigrees was observed most often: in CG - 81.3\%; DG1 - 91.1\%; DG2 - $99 \%$ (Fig. 1).

Let's analyze the burden of pedigrees of patients of all groups with somatic, psychoneurological and oncological diseases. The identified cases of somatic pathology belonged to diseases of the urinary, gastrointestinal, endocrine, cardiovascular and respiratory systems. The results of comparing the incidence rate are shown in Fig. 2 and in Table 2. A high proportion of cardiovascular, oncological and psychoneurological pathology in the pedigrees of patients of all groups was found. Pathology of the cardiovascular system was recorded in CG - 34.38\%, DG1 - 46.43\%, DG2 - $97.96 \%$; oncological diseases in CG - 31.25 \%, DG1 - 42.86 \%, DG2 - 79.59 \%; psychoneurological diseases in CG $-21.88 \%$, DG1 - 37.5\%, DG2 - $44.9 \%$; diseases of the gastrointestinal tract in CG - $21.88 \%$, DG1 - 23.21\%, DG2 - $27.55 \%$; endocrine diseases in CG - $18.75 \%$, DG1 $17.86 \%$, DG2 - $22.45 \%$; respiratory diseases in CG - $12.5 \%$, DG1 - 16.07 \%, DG2 - $7.14 \%$; diseases of the urinary system in CG $-12.5 \%$, DG1 - 8.93\%, DG2 - $11.22 \%$. All types of pathology were more common in relatives of DG2 patients compared to DG1: cardiovascular system - by $51.53 \%$; oncological - by $36.73 \%$; psychoneurological - by $7.40 \%$; endocrine - by $4.59 \%$; gastrointestinal tract - by $4.34 \%$ and urinary system - by $2.29 \%$. 


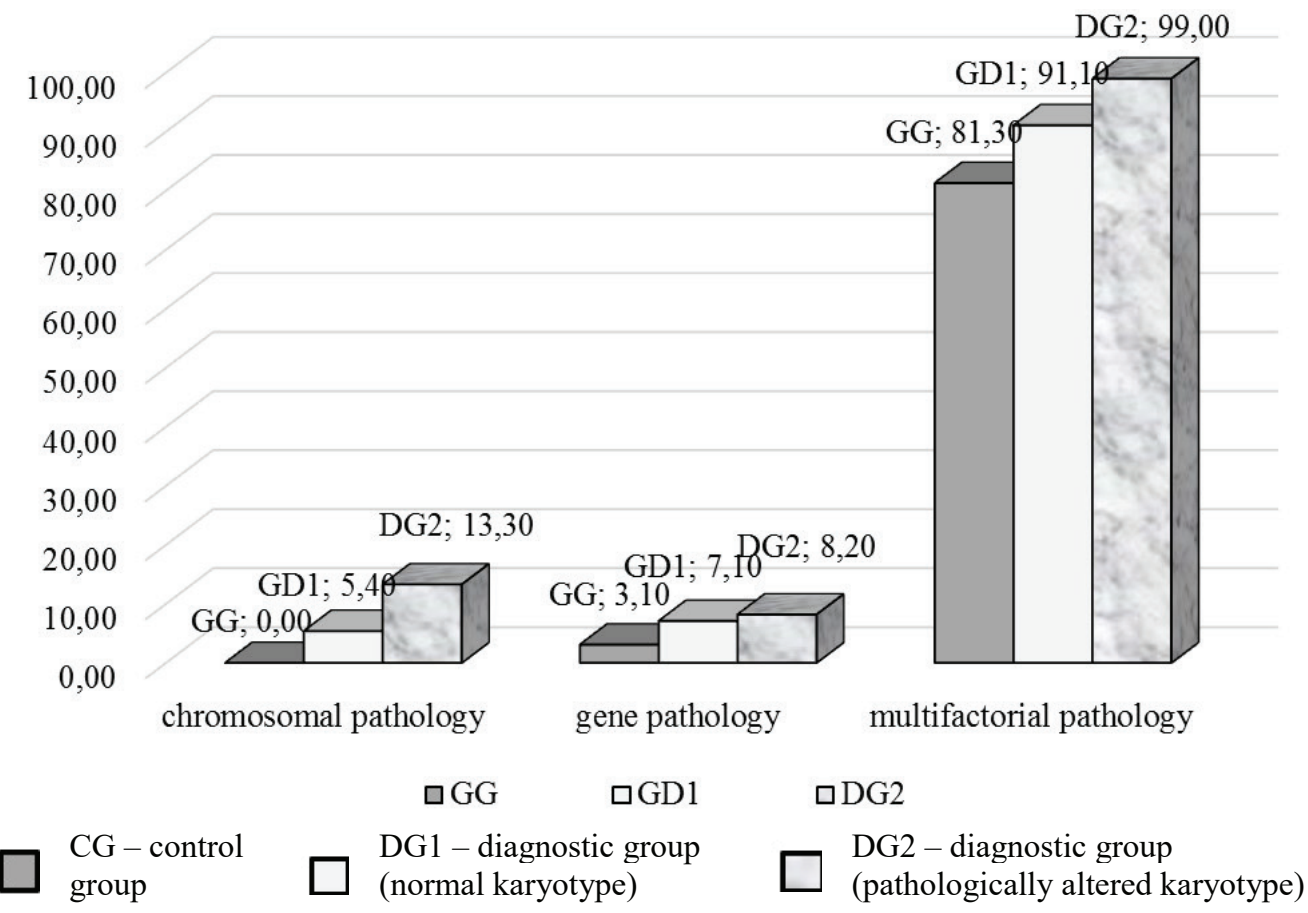

Fig. 1. The burden of pedigree examined patients with DG and CG

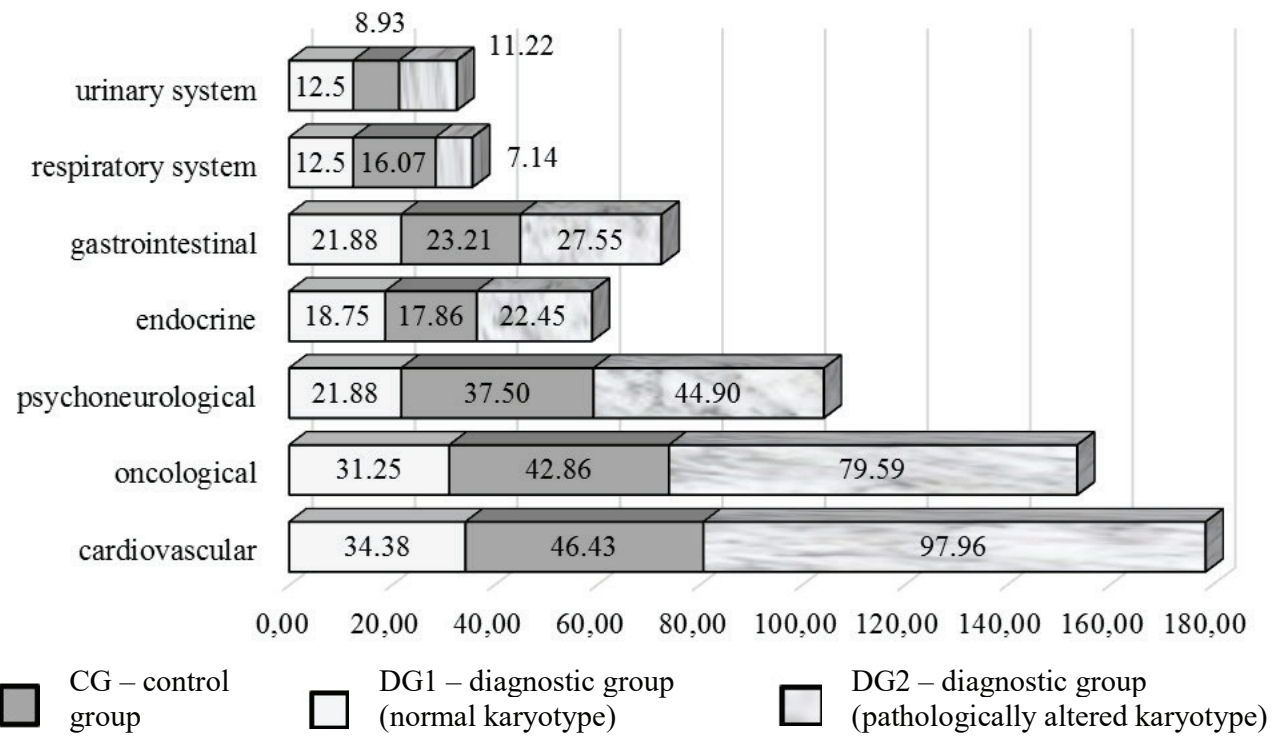

Fig. 2. Structural characteristics of multifactorial pathology in the pedigrees of the examined patients

The data in Table 2 show that the relatives of DG2 patients were most common: varicose veins (26.53\%), myocardial infarction (21.43\%), strokes (16.33\%), gastric and lung cancer (12.24 \% each), mammary cancer $(10.2 \%)$, alcohol dependence and diabetes mellitus (12.24\% each), pancreatitis $(8.16 \%)$. The predominance of cardiovascular, oncological and psychoneurological pathology in the pedigree of patients in this group corresponds to the predominance of organ's systems lesions in fetus and newborns with birth defects. This proves the connection with the carrier and inheritance of mutant (polymorphic) alleles of the genes of the FC system, which are responsible for such malformations. The world scientific community has studied in detail the dependence of the functionality of FC genes in cases of MTHFR and MTRR gene polymorphism on the type of their inheritance (homozygous and heterozygous), according to which the homozygous type of gene polymorphism inheritance reduces its functionality by approximately $70 \%$ and heterozygous $35 \%[9,10]$. 
Table 2

Frequency and structural characteristics of pathology in the pedigrees of the surveyed families of diagnostic and control groups (absolute amount; \%)

\begin{tabular}{|c|c|c|c|c|c|c|}
\hline \multirow{2}{*}{ System, pathology } & \multicolumn{2}{|c|}{ DG1 $(n=56)$} & \multicolumn{2}{|c|}{ DG2 $(n=98)$} & \multicolumn{2}{|c|}{ CG $(n=32)$} \\
\hline & abs. & $\%$ & abs. & $\%$ & abs. & $\%$ \\
\hline \multicolumn{7}{|c|}{ pathology of the cardiovascular system } \\
\hline Hereditary thrombophilia & 2 & 3.57 & 8 & 8.16 & - & - \\
\hline Myocardial infarction & 3 & 5.36 & 21 & 21.43 & 2 & 6.25 \\
\hline Ischemic heart disease, stenocardia & 7 & 12.50 & 17 & 17.35 & 3 & 9.38 \\
\hline Varicose veins & 4 & 7.14 & 26 & 26.53 & 2 & 6.25 \\
\hline Hypertension & 8 & 14.29 & 16 & 16.33 & 3 & 9.38 \\
\hline Congenital heart disease & 1 & 1.79 & 4 & 4.08 & - & - \\
\hline Atherosclerosis & 1 & 1.79 & 3 & 3.06 & 1 & 3.13 \\
\hline Total & 26 & 46.43 & 96 & 97.96 & 11 & 34.38 \\
\hline \multicolumn{7}{|c|}{ oncological pathology } \\
\hline Gastric cancer & 4 & 7.14 & 12 & 12.24 & 2 & 6.25 \\
\hline Bowel cancer & 2 & 3.57 & 6 & 6.12 & - & - \\
\hline Lung cancer & 2 & 3.57 & 12 & 12.24 & 2 & 6.25 \\
\hline Laryngeal cancer & 2 & 3.57 & 3 & 3.06 & - & - \\
\hline Mammary cancer & 4 & 7.14 & 10 & 10.20 & 1 & 3.13 \\
\hline Bladder Cancer & 1 & 1.79 & 2 & 2.04 & - & - \\
\hline Skin cancer & - & - & 2 & 2.04 & - & - \\
\hline Prostate adenoma & - & - & 5 & 5.10 & 1 & 3.13 \\
\hline Benign uterine tumor & 3 & 5.36 & 12 & 12.24 & 2 & 6.25 \\
\hline Benign ovarian tumor & 6 & 10.71 & 14 & 14.29 & 2 & 6.25 \\
\hline Total & 24 & 42.86 & 78 & 79.59 & 10 & 31.25 \\
\hline \multicolumn{7}{|c|}{ psychoneurological pathology } \\
\hline Stroke & 6 & 10.71 & 16 & 16.33 & 4 & 12.50 \\
\hline Schizophrenia & 2 & 3.57 & 5 & 5.10 & 1 & 3.13 \\
\hline Alcoholism & 5 & 8.93 & 12 & 12.24 & 1 & 3.13 \\
\hline Epilepsy & 4 & 7.14 & 6 & 6.12 & - & - \\
\hline Migraine & 1 & 1.79 & 2 & 2.04 & 1 & 3.13 \\
\hline Developmental delay & 3 & 5.36 & 3 & 3.06 & - & - \\
\hline Total & 21 & 37.50 & 44 & 44.90 & 7 & 21.88 \\
\hline \multicolumn{7}{|c|}{ endocrine pathology } \\
\hline Diabetes mellitus & 5 & 8.93 & 12 & 12.24 & 3 & 9.38 \\
\hline Obesity & 2 & 3.57 & 6 & 6.12 & 2 & 6.25 \\
\hline Autoimmune thyroiditis & 3 & 5.36 & 4 & 4.08 & 1 & 3.13 \\
\hline Total & 10 & 17.86 & 22 & 22.45 & 6 & 18.75 \\
\hline \multicolumn{7}{|c|}{ pathology of the gastrointestinal tract } \\
\hline Gastroduodenitis & 3 & 5.36 & 5 & 5.10 & 2 & 6.25 \\
\hline Peptic ulcer & 4 & 7.14 & 7 & 7.14 & 2 & 6.25 \\
\hline Pancreatitis & 2 & 3.57 & 8 & 8.16 & - & - \\
\hline Gallstone disease & 3 & 5.36 & 5 & 5.10 & 3 & 9.38 \\
\hline Liver Cirrhosis & 1 & 1.79 & 2 & 2.04 & - & - \\
\hline Total & 13 & 23.21 & 27 & 27.55 & 7 & 21.88 \\
\hline \multicolumn{7}{|c|}{ respiratory pathology } \\
\hline Chronic bronchitis & 4 & 7.14 & 3 & 3.06 & 2 & 6.25 \\
\hline Bronchial asthma & 3 & 5.36 & 2 & 2.04 & 1 & 3.13 \\
\hline Chronic sinusitis & 2 & 3.57 & 2 & 2.04 & 1 & 3.13 \\
\hline Total & 9 & 16.07 & 7 & 7.14 & 4 & 12.50 \\
\hline \multicolumn{7}{|c|}{ pathology of the urinary system } \\
\hline Urolithiasis & 3 & 5.36 & 4 & 4.08 & 2 & 6.25 \\
\hline Chronic pyelonephritis & 2 & 3.57 & 7 & 7.14 & 2 & 6.25 \\
\hline Total & 5 & 8.93 & 11 & 11.22 & 4 & 12.50 \\
\hline
\end{tabular}


In a retrospective study of the relationship between polymorphism of genes encoding the enzymes FC (C677T MTHFR and A66G MTRR), malformations and miscarriages diagnosed in 6824 clinical cases for the period 2008-2013. A linear logistic regression calculation for different configurations of homozygous and heterozygous gene disorders in these patients were performed (Table 3). Comparisons were made with patients without polymorphisms of these genes. The group of patients with heterozygous type of MTHFR and MTRR genes polymorphism (named MTHFR1 and MTRR1) had "milder" consequences of this disorder (less reduction in genes functionality). The group of patients with homozygous type of MTHFR and MTRR genes polymorphism (named MTHFR2 and MTRR2), accordingly, had "more severe" consequences (greater reduction in genes functionality). This result of the study, which was partially discussed in [11], corresponds to the data of other researchers $[12,13]$. The groups MTHFR1 and MTHFR2, $M T R R 1$ and MTRR2 were compared with the groups of patients who did not record polymorphisms of the MTHFR and MTRR genes (named MTHFRO and MTRRO). RL in anamnesis of patients $M T H F R O$ and $M T R R O$ were associated with other risk factors: primarily smoking, alcohol and drug use, psycho-emotional overload, the negative effects of harmful environmental and/or industrial factors, etc.

Table 3

Association of clinically manifested FC disorders with established polymorphic genes MTHFR and MTRR of hetero- and homozygous types of inheritance in patients with anamnestic confirmed RL, examined for the period 2008-2013 in MNCE KhRC "IRSMGC-CR(O)D"

\begin{tabular}{|c|c|c|c|c|c|c|c|}
\hline \multirow{2}{*}{ Indicators } & \multicolumn{2}{|c|}{ Non-standardized coefficients } & \multirow{2}{*}{$\frac{\text { Standardized coefficients }}{\text { Beta }}$} & \multirow{2}{*}{$t$} & \multirow{2}{*}{$p$} & \multicolumn{2}{|c|}{$95.0 \%$ TI } \\
\hline & $B$ & $m$ & & & & lower & upper \\
\hline Constanta & 0.035 & 0.004 & - & 8.522 & 0.000 & 0.027 & 0.043 \\
\hline MTHFR1 & 0.999 & 0.004 & 0.359 & 280.396 & 0.000 & 0.992 & 1.006 \\
\hline MTHFR2 & 2.675 & 0.006 & 0.578 & 452.214 & 0.000 & 2.663 & 2.686 \\
\hline MTRR1 & 0.998 & 0.004 & 0.361 & 224.232 & 0.000 & 0.989 & 1.006 \\
\hline MTRR2 & 2.902 & 0.005 & 0.996 & 619.270 & 0.000 & 2.893 & 2.911 \\
\hline
\end{tabular}

Note: $t$ - $t$-statistics, $p$ - statistical significance of the difference

The study (Table 3) showed that compared with the group of patients without confirmed polymorphism of MTHFR gene (MTHFRO) in subjects with heterozygous inheritance (MTHFRI) probably $(p=0.00)$ and significantly increased the chances of RL (by 0.999 units, or 29.5 times); and at homozygous significantly ( $p=0.000$; by 2,675 units, or 77.43 times). Similarly, compared with the group of patients without confirmed polymorphism of the MTRR gene (MTRRO) significantly ( $p=0.000$ ) increased the chances: in heterozygous inheritance (MTRRI) by 0.998 units, or 29.51 times; when homozygous by 2,902 units, or 83.91 times.

Our data on the probable relationships of polymorphism of genes encoding FC (C677T MTHFR and A66G MTRR) and responsible for most fetal malformations and RL in general, were fully confirmed by correlation-regression analysis. Groups of homo- and heterozygous polymorphisms of the C677T MTHFR and A66G MTRR genes (MTHFR1; MTHFR2; MTRR1 and $M T R R 2)$ were studied in this investigation. Hypothesis about correlation between the degree of polymorphism C677T MTHFR and A66G MTRR genes with the "severity" of FC excitation in the range from "mild" (heterozygous polymorphism of one of the two genes) to quite "severe" (homozygous polymorphism for both genes) was proven and confirmed in variable series: polymorphism of heterozygous type isolated by one gene; - polymorphism of heterozygous type by two genes; - polymorphism of homozygous type isolated by one gene; - polymorphism of homozygous type for one gene and the second heterozygous type; - polymorphism of homozygous type by two genes (Table 4).

The degree of gene polymorphism probably correlated by moderate force with the presence of MTHFR gene polymorphism $(r=0.542 ; p=0.000)$ and by high force with MTRR gene polymorphism $(r=0.798 ; p=0.000)$. In this case, it was determined that the degree of polymorphism has a probable weak relationship with heterozygous polymorphism of the genes MTHFR $(r=0.222$; $p=0.000)$ and $\operatorname{MTRR}(r=-0.259 ; p=0.000)$, which provoke "milder" disorders of FC and, respectively, responsible for smaller RL. In contrast, homozygous gene polymorphism is likely and strong- 
ly associated with "severe" FC disorders, which are responsible for greater RL risks: MTHFR $(r=0.432 ; p=0.000)$ and $\operatorname{MTRR}(r=0.747 ; p=0.000)$.

Table 4

Correlation of clinically manifested FC disorders with polymorphic genes MTHFR and MTRR of hetero- and homozygous inheritance types of and with the degree of their polymorphism in patients with anamnestic confirmed RL (examined in 2008-2013 in MNCE KhRC "IRSMGC-CR(O)D”)

\begin{tabular}{ccccccccc}
\hline Genes & & MTHFR & MTRR & Degree of polymorphism & MTHFR1 & MTHFR2 & MTRRI & MTRR2 \\
\hline \multirow{2}{*}{ MTHFR } & $r$ & 1.000 & -0.011 & $0.542^{* *}$ & $0.624^{* *}$ & $0.572^{* *}$ & 0.012 & -0.014 \\
& $p$ & - & 0.370 & 0.000 & 0.000 & 0.000 & 0.305 & 0.233 \\
& $n$ & 6824 & 6824 & 6824 & 6824 & 6824 & 6824 & 6824 \\
& $r$ & -0.011 & 1.000 & $0.798^{* *}$ & -0.001 & -0.013 & $-0.212^{* *}$ & $0.882^{* *}$ \\
MTRR & $p$ & 0.370 & - & 0.000 & 0.950 & 0.301 & 0.000 & 0.000 \\
& $n$ & 6824 & 6824 & 6824 & 6824 & 6824 & 6824 & 6824 \\
Degree of & $r$ & $0.542^{* *}$ & $0.798^{* *}$ & 1.000 & $0.222^{* *}$ & $0.432^{* *}$ & $-0.259^{* *}$ & $0.747^{* *}$ \\
polymor- & $p$ & 0.000 & 0.000 & - & 0.000 & 0.000 & 0.000 & 0.000 \\
phism & $n$ & 6824 & 6824 & 6824 & 6824 & 6824 & 6824 & 6824 \\
& $r$ & $0.624^{* *}$ & -0.001 & $0.222^{* *}$ & 1.000 & $-0.284^{* *}$ & 0.016 & -0.008 \\
MTHFR1 & $p$ & 0.000 & 0.950 & 0.000 & - & 0.000 & 0.192 & 0.498 \\
& $n$ & 6824 & 6824 & 6824 & 6824 & 6824 & 6824 & 6824 \\
& $r$ & $0.572^{* *}$ & -0.013 & $0.432^{* *}$ & $-0.284^{* *}$ & 1.000 & -0.001 & -0.009 \\
MTHFR2 & $p$ & 0.000 & 0.301 & 0.000 & 0.000 & - & 0.912 & 0.451 \\
& $n$ & 6824 & 6824 & 6824 & 6824 & 6824 & 6824 & 6824 \\
& $r$ & 0.012 & $-0.212^{* *}$ & $-0.259^{* *}$ & 0.016 & -0.001 & 1.000 & $-0.647^{* *}$ \\
MTRR1 & $p$ & 0.305 & 0.000 & 0.000 & 0.192 & 0.912 & - & 0.000 \\
& $n$ & 6824 & 6824 & 6824 & 6824 & 6824 & 6824 & 6824 \\
& $r$ & -0.014 & $0.882^{* *}$ & $0.747^{* *}$ & -0.008 & -0.009 & $-0.647^{* *}$ & 1.000
\end{tabular}

Note: $r$-correlation coefficient; $p$-statistical significance of the difference; $n$-total number of observations; ${ }^{* *}-r e-$ liable relationship

\section{Discussion}

Our results on the greater dependence of the FC genes functionality of in cases of polymorphism MTHFR and MTRR genes by the type of their inheritance (homozygous and heterozygous) completely coincide with other studies. Thus, according to Greenberg J. A. et al. [11], the homozygous type of inheritance of gene polymorphism reduces its functionality by $70 \%$, and heterozygous - by approximately $35 \%$. Other studies have also shown a higher effect on the homozygous type of inheritance of these genes in FC disorders $[12,13]$.

However, there are no literary sources that would describe exactly how these observations affect the organization of medical care for patients with a burdened obstetric anamnesis. Determination of the type of inheritance of FC polymorphic genes makes it possible to collect statistics on the severity of clinical manifestations and build predictive models about the number of pregnancies with unfavorable outcomes, the number of disabled children of varying severity. A woman who has given birth to a child with a disability (birth defects) is virtually excluded from the number of women of reproductive age who will be planning another pregnancy. Naturally, the quality of life of the family in which the disabled child is growing will also be reduced. Our study also connects for the first time two such important aspects of genetically determined RL, such as the severity of the clinical manifestations of FC disorders and the possible necessary actions of medical services in preconception care for the next pregnancy.

The fact proved by us for the population of the Kharkiv region of Ukraine allows to plan budget expenditures for health care, taking into account the known annual number of miscarriages and congenital malformations of fetuses and newborns, as well as the estimated number of clinically manifested cases of FC disorders of varying severity. A woman in whom 
a violation of the folate cycle could not be detected in the early stages of embryo and fetal development, who has the most severe variant of the development of a congenital defect (homozygous polymorphism, MTHFR2 and MTRR2), should be able to terminate the pregnancy both before 15 weeks of pregnancy, as and the right to a late abortion. Understanding the risk of the most severe clinical manifestations for the entire population of such patients allows discussing legislative changes on abortion issues.

All medical specialists (family doctors, pediatricians, obstetricians-gynecologists, pediatric surgeons) should be notified of the possibility of diagnosing the severity of clinical manifestations of folate cycle disorders. Their actions for early diagnosis and the necessary medication and dietary intervention can lead to a significant reduction in reproductive losses in both the Kharkov region and Ukraine.

Study limitations. Significant financial costs due to the high cost of genetic testing.

Prospects for further research. Further research is planned to determine the medical and social possibilities of optimizing the model of prevention of genetically determined reproductive losses.

\section{Conclusions}

1. When studying the influence of medical-genetic risk factors to the level of anamnestic confirmed in-depth studied causes of genetically determined RL as a systemic phenomenon was validate. Structural characteristics of RL were stated, most of which are combinations of different manifestations, or conditions and diseases such as: missed abortions, miscarriages, antenatal fetal death, ectopic pregnancies, secondary infertility, early neonatal fetal death and multiple intrauterine fetal malformations (birth defects). Genetically determined RL are primarily associated with miscarriage and congenital malformations of embryo, fetus and newborn. The most significant genetic factor of miscarriage and birth defects is FC genes polymorphism, which can be determined both by direct genetic tests and indirectly by impaired blood coagulation system parameters and amino acid metabolism (primarily homocysteine).

2. The high influence of hereditary medical-genetic factors on the increased risks of RL for population has been clarified. A significant predominance of all types pathology (chromosomal, gene and multifactorial) in the pedigrees of those examined with a burdensome RL obstetric anamnesis of (compared with control, patients without anamnestical burdens) was found. First of all, this manifested itself in the form of cardiovascular, oncological and psychoneurological pathology among relatives of patients with RL, and the frequency of occurrence depended on the karyotype.

3. The risk of reproductive loss is reliably associated with the type of inheritance of MTHFR and MTRR polymorphic genes: the most "severe" homozygous type of polymorphism of both genes, the least severe - the heterozygous monogenic type. Diagnosis of gene polymorphism allows timely correction of the condition with the help of vitamin therapy and diet, to make an informed decision on maintaining or terminating pregnancy. Determining the possible severity of clinical manifestations makes it possible to more accurately determine the RL risks.

\section{Conflict of interests}

The authors declare that they have no conflicts of interest.

\section{References}

[1] Shi, T., Huang, L.-J., Xiong, Y.-Q., Zhong, Y.-Y., Yang, J.-J., Fu, T. et. al. (2018). The risk of herpes simplex virus and human cytomegalovirus infection during pregnancy upon adverse pregnancy outcomes: A meta-analysis. Journal of Clinical Virology, 104, 48-55. doi: http://doi.org/10.1016/j.jcv.2018.04.016

[2] Pinar, M. H., Gibbins, K., He, M., Kostadinov, S., Silver, R. (2018). Early Pregnancy Losses: Review of Nomenclature, Histopathology, and Possible Etiologies. Fetal and Pediatric Pathology, 37 (3), 191-209. doi: http://doi.org/10.1080/15513815.2018.1455775

[3] Shahine, L., Lathi, R. (2015). Recurrent Pregnancy Loss. Obstetrics and Gynecology Clinics of North America, 42 (1), $117-134$. doi: http://doi.org/10.1016/j.ogc.2014.10.002 
[4] Kohn, T. P., Kohn, J. R., Darilek, S., Ramasamy, R., Lipshultz, L. (2016). Genetic counseling for men with recurrent pregnancy loss or recurrent implantation failure due to abnormal sperm chromosomal aneuploidy. Journal of Assisted Reproduction and Genetics, 33 (5), 571-576. doi: http://doi.org/10.1007/s10815-016-0702-8

[5] Robinson, G. E. (2014). Pregnancy loss. Best Practice \& Research Clinical Obstetrics \& Gynaecology, 28 (1), 169-178. doi: http://doi.org/10.1016/j.bpobgyn.2013.08.012

[6] Romero, S. T., Geiersbach, K. B., Paxton, C. N., Rose, N. C., Schisterman, E. F., Branch, D. W., Silver, R. M. (2015). Differentiation of genetic abnormalities in early pregnancy loss. Ultrasound in Obstetrics \& Gynecology, 45 (1), 89-94. doi: http://doi.org/10.1002/uog.14713

[7] Ouyang, Y., Tan, Y., Yi, Y., Gong, F., Lin, G., Li, X., Lu, G. (2016). Correlation between chromosomal distribution and embryonic findings on ultrasound in early pregnancy loss after IVF-embryo transfer. Human reproduction, 31 (10), $2212-2218$. doi: http://doi.org/10.1093/humrep/dew201

[8] Yi, Y., Lu, G., Ouyang, Y., lin, G., Gong, F., Li, X. (2016). A logistic model to predict early pregnancy loss following in vitro fertilization based on 2601 infertility patients. Reproductive Biology and Endocrinology, 14 (1). doi: http://doi.org/10.1186/ s12958-016-0147-z

[9] Waterman, C., Batstone, P., Bown, N., Cresswell, L., Delmege, C., English, C. et. al. (2016). The clinical utility of genetic testing of tissues from pregnancy losses. BJOG: An International Journal of Obstetrics \& Gynaecology, 125 (7), $867-873$. doi: http://doi.org/10.1111/1471-0528.14229

[10] Kacprzak, M., Chrzanowska, M., Skoczylas, B., Moczulska, H., Borowiec, M., Sieroszewski, P. (2016). Genetic causes of recurrent miscarriages. Ginekologia Polska, 87 (10), 722-726. doi: http://doi.org/10.5603/gp.2016.0075

[11] Greenberg, J. A., Bell, S. J., Guan, Y., Yu, Y. H. (2011). Folic Acid supplementation and pregnancy: more than just neural tube defect prevention. Reviews in Obstetrics \& Gynecology, 4 (2), 52-59.

[12] Scazziota, A., Pons, S., Raimondi, R., Fernandez, C. (2000). Is C677T mutation in the methylenetetrahydrofolate reductase (MTHFR) a risk factor for arterial thrombosis? 16th Congress oh thrombosis and haemostasis. Porto, 47-53.

[13] Grechanina, E. Ia., Lesovoi, V. N., Miasoedov, V. V., Grechanina, Iu. B., Gusar, V. A. (2010). Zakonomernaia sviaz mezhdu razvitiem nekotorykh epigeneticheskikh boleznei i narusheniem metilirovaniia DNK vsledstvie defitsita fermentov folatnogo tsikla. Ultrazvukova perinatalna diagnostika, 29, 27-59. Available at: http://repo.knmu.edu.ua/handle/123456789/628 